\title{
Correction to: Services according to mental health needs for youth in foster care? - a multi-informant study
}

Marit Larsen ${ }^{*}$, Valborg Baste ${ }^{2}$, Ragnhild Bjørknes ${ }^{3}$, Trine Myrvold ${ }^{4}$ and Stine Lehmann ${ }^{1}$

\section{Correction}

Following publication of the original article [1], the authors reported a misaligning of data in Table 3 (Table 1 here) (weekly results have been put in the column for monthly results).

The correct Table 3 (Table 2 here) is shown below.

The original publication of this article has been corrected.

\begin{abstract}
Author details
'Regional Centre for Child and Youth Mental Health and Child Welfare West, Uni Research Health, Bergen, Norway. ${ }^{2}$ Uni Research Health, Bergen, Norway. ${ }^{3}$ Department of Health Promotion and Development, Faculty of Psychology, University of Bergen, Bergen, Norway. ${ }^{4}$ The Norwegian Institute for Urban and Regional Research, Oslo Metropolitan University, Oslo, Norway.
\end{abstract}

Received: 27 August 2018 Accepted: 27 August 2018

Published online: 11 September 2018

\section{Reference}

1. Larsen et al. (2018) Services according to mental health needs for youth in foster care? - A multi-informant study (2018) 18:63a https://doi.org/10.1186/ s12913-018-3365-6.

\footnotetext{
*Correspondence: marit.larsen@uni.no

${ }^{1}$ Regional Centre for Child and Youth Mental Health and Child Welfare -

West, Uni Research Health, Bergen, Norway
} 
Table 1 Service contact reported by carers $(n=330)$ and youths $(n=224)$

\begin{tabular}{|c|c|c|c|c|c|c|}
\hline & \multirow{2}{*}{$\begin{array}{l}\text { Serviles } \\
\text { Cantect }\end{array}$} & \multicolumn{5}{|c|}{ Ditriflipution af use fire the ones that have had contact } \\
\hline & & $\begin{array}{l}\text { Evary wook } \\
\text { (1) }\end{array}$ & \multicolumn{2}{|c|}{ Evary manth (D) } & $\begin{array}{l}\text { Evory } 3 . \\
\text { Month (X) }\end{array}$ & $\begin{array}{l}\text { Evary } 6 . \\
\text { Manth (1) }\end{array}$ \\
\hline & $5(\mathrm{~m})$ & $5(m)$ & \multicolumn{2}{|l|}{$\%(n)$} & $6(n)$ & 50 \\
\hline \multicolumn{7}{|l|}{ CAMHS } \\
\hline Caners & $31.2(103)$ & $233(24)$ & \multicolumn{2}{|l|}{43.7 (45) } & $13.5(14)$ & $19.4(20)$ \\
\hline Fouth & $27.2(61)$ & 18.0 (111) & \multicolumn{2}{|l|}{$44.3(27)$} & $16.4(10)$ & $213(13)$ \\
\hline \multicolumn{7}{|c|}{ Contact with Frimary Halth Cars sarvices: } \\
\hline Caners & $61.2(202)$ & & & & & \\
\hline Fouth & $385(131)$ & & & & & \\
\hline \multicolumn{7}{|c|}{ Sthoul Halth service } \\
\hline Carwrs & $27.5(91)$ & & $3.9(9)$ & $\begin{array}{l}20.9 \\
(19)\end{array}$ & $28.6(26)$ & $45.1(41)$ \\
\hline Youth & $30.8(69)$ & & $\begin{array}{l}14.9 \\
(10)\end{array}$ & $\begin{array}{l}19.9 \\
\text { (11) }\end{array}$ & $33.3(23)$ & $36.2(23)$ \\
\hline \multicolumn{7}{|c|}{ Educatienal Psychalagy Sartice } \\
\hline Carwrs & 34.8 (11.15) & & $5.2(6)$ & $\begin{array}{l}10.4 \\
(127)\end{array}$ & $34.8(-6)$ & $49.5(37)$ \\
\hline routh & $19.6(4)$ & & $11.4(5)$ & $\begin{array}{l}27.3 \\
(12)\end{array}$ & $19.9(7)$ & $43.5(20)$ \\
\hline \multicolumn{7}{|c|}{ Cenaral Fractitioner } \\
\hline Cansrs & 29.7 (98) & & $1.0(1)$ & $6.1(5)$ & $27.5(27)$ & $63.3(64)$ \\
\hline Fouth & $35.7(80)$ & & $2.5(2)$ & $\begin{array}{l}10.0 \\
(8)\end{array}$ & $30.0(24)$ & $37.3(46)$ \\
\hline \multicolumn{7}{|c|}{ Adolsewnt Hoalth Girse } \\
\hline Caners & $73(24)$ & & $0(0)$ & $\begin{array}{l}20.8 \\
\text { (5) }\end{array}$ & $8.3(2)$ & $70.8(17)$ \\
\hline Fouth & $11.6(26)$ & & $3.9(1)$ & $\begin{array}{l}11.3 \\
(3)\end{array}$ & 19.2 (5) & $65.4(17)$ \\
\hline \multicolumn{7}{|c|}{ Other sarvios providars: } \\
\hline \multicolumn{7}{|c|}{ Murdeipal Cos } \\
\hline Carwrs & $921(904)$ & & $23(7)$ & $\begin{array}{l}20.4 \\
(62)\end{array}$ & $54.9(157)$ & $22.4(68)$ \\
\hline Fouth & $85.3(191)$ & & $1.0(7)$ & $\begin{array}{l}7.9 \\
(15)\end{array}$ & $52.4(100)$ & $38.7(74)$ \\
\hline \multicolumn{7}{|c|}{ Special Education } \\
\hline Garwers & $427(141)$ & & $\begin{array}{l}77.3 \\
(109)\end{array}$ & $\begin{array}{l}9.2 \\
(1.3)\end{array}$ & $3.6(5)$ & $9.9(14)$ \\
\hline routh & 21.9 (49) & & $\begin{array}{l}66.3 \\
(32)\end{array}$ & $\begin{array}{l}12.2 \\
(6)\end{array}$ & $12.2(6)$ & $10.2(3)$ \\
\hline \multicolumn{7}{|c|}{ Othar sarvites } \\
\hline Carwrs & $15.4(54)$ & & $\begin{array}{l}22.2 \\
(12)\end{array}$ & $\begin{array}{l}38.9 \\
\text { (21) }\end{array}$ & $22.2(12)$ & $15.7(9)$ \\
\hline Fouth & $7.1(16)$ & & $18.8(3)$ & $\begin{array}{l}31.3 \\
\text { (5) }\end{array}$ & $25.0(0)$ & $23.0(4)$ \\
\hline
\end{tabular}


Table 2 Service contact reported by carers $(n=330)$ and youths $(n=224)$

\begin{tabular}{|c|c|c|c|c|c|}
\hline & \multirow{3}{*}{$\begin{array}{l}\text { Service contact } \\
\%(n)\end{array}$} & \multicolumn{4}{|c|}{ Distribution of use for the ones that have had contact. } \\
\hline & & Every week (4) & Every month (3) & Every 3. month (2) & Every 6. month (1) \\
\hline & & $\%(n)$ & $\%(n)$ & $\%(n)$ & $\%(n)$ \\
\hline \multicolumn{6}{|l|}{ CAMHS } \\
\hline Carers & $31.2(103)$ & $23.3(24)$ & $43.7(45)$ & $13.6(14)$ & $19.4(20)$ \\
\hline Youth & $27.2(61)$ & $18.0(11)$ & $44.3(27)$ & $16.4(10)$ & $21.3(13)$ \\
\hline \multicolumn{6}{|c|}{ Contact with primary health care services: } \\
\hline Carers & $61.2(202)$ & & & & \\
\hline Youth & $58.5(131)$ & & & & \\
\hline \multicolumn{6}{|c|}{ School health service } \\
\hline Carers & $27.6(91)$ & $5.5(5)$ & $20.9(19)$ & $28.6(26)$ & $45.1(41)$ \\
\hline Youth & $30.8(69)$ & $14.5(10)$ & $15.9(11)$ & $33.3(23)$ & $36.2(25)$ \\
\hline \multicolumn{6}{|c|}{ Educational psychology service } \\
\hline Carers & $34.8(115)$ & $5.2(6)$ & $10.4(12)$ & $34.8(40)$ & $49.6(57)$ \\
\hline Youth & $19.6(44)$ & $11.4(5)$ & $27.3(12)$ & $15.9(7)$ & $45.5(20)$ \\
\hline \multicolumn{6}{|c|}{ General Practitioner } \\
\hline Carers & $29.7(98)$ & $1.0(1)$ & $6.1(6)$ & $27.6(27)$ & $65.3(64)$ \\
\hline Youth & $35.7(80)$ & $2.5(2)$ & $10.0(8)$ & $30.0(24)$ & $57.5(46)$ \\
\hline \multicolumn{6}{|c|}{ Adolescent health clinic } \\
\hline Carers & $7.3(24)$ & $0(0)$ & $20.8(5)$ & $8.3(2)$ & $70.8(17)$ \\
\hline Youth & $11.6(26)$ & $3.9(1)$ & $11.5(3)$ & $19.2(5)$ & $65.4(17)$ \\
\hline \multicolumn{6}{|c|}{ Other service providers: } \\
\hline \multicolumn{6}{|c|}{ Municipal CPS } \\
\hline Carers & $92.1(304)$ & $2.3(7)$ & $20.4(62)$ & $54.9(167)$ & $22.4(68)$ \\
\hline Youth & $85.3(191)$ & $1.0(2)$ & $7.9(15)$ & $52.4(100)$ & 38.7 (74) \\
\hline \multicolumn{6}{|c|}{ Special Education } \\
\hline Carers & $42.7(141)$ & $77.3(109)$ & $9.2(13)$ & $3.6(5)$ & $9.9(14)$ \\
\hline Youth & $21.9(49)$ & $65.3(32)$ & $12.2(6)$ & $12.2(6)$ & $10.2(5)$ \\
\hline \multicolumn{6}{|c|}{ Other services } \\
\hline Carers & $16.4(54)$ & $22.2(12)$ & 38.9 (21) & $22.2(12)$ & $16.7(9)$ \\
\hline Youth & $7.1(16)$ & $18.8(3)$ & $31.3(5)$ & $25.0(4)$ & $25.0(4)$ \\
\hline
\end{tabular}

